\title{
A TOLERANCE ANALYSIS AND OPTIMIZATION METHODOLOGY. THE COMBINED USE OF 3D CAT, A DIMENSIONAL HIERARCHIZATION MATRIX AND AN OPTIMIZATION ALGORITHM
}

\author{
Basilio Ramos Barbero ${ }^{1}$, Jorge Pérez Azcona ${ }^{2}$, Jorge González Pérez ${ }^{2}$ \\ ${ }^{1}$ Graphic Expression Department, Escuela Politécnica Superior, Universidad de Burgos, \\ Avda. Cantabria s/n. 09006 Burgos, Spain. \\ 2 Antolin-Group, Ctra, Madrid-Irún, Km 244.8, Burgos, Spain.
}

\begin{abstract}
We propose a methodology in this study for the analysis and the optimization of assembly tolerances. A combination of three components, it involves the use of 3D CAT software, a table referred to as a "dimensional hierarchization matrix", and a tolerance optimization algorithm. The Antolin Group, a Spanish multinational in the automobile components sector, employs this system to optimize tolerance values and to reduce manufacturing costs. The matrix was designed to enable easy identification, in a single table, of all requirements that fail to meet the specifications in the different approximations, prior to the definition of the dimensional and the geometric tolerances that comply with the functional requirements, and to identify which tolerances contribute most to variations in all of the functional conditions of the mechanism. Through its different iterations, this matrix allows us to see which of the tolerances should first be modified to optimize the design requirement specifications. A tolerance optimization algorithm was also defined, which functions with the data from the dimensional hierarchization matrix.
\end{abstract}

Keywords: tolerance allocation; tolerance analysis; functional dimensioning and tolerancing; 3D CAT; 3D assembly; tolerance optimization

\section{Introduction.}

Tolerance analysis evaluates dimensional and/or geometric variations in an assembly on the basis of a stack of dimensions and tolerances that may be applied to its components. Tolerance analysis is a fundamental tool in the definition of the tolerances for single components, solving the trade-off between product quality and cost.

Unfortunately, designers have only two types of tools to perform tolerance analyses, which neither simulate the real behaviour of parts or joints in the mechanisms, nor imply compliance with ASME and ISO standards that refer to tolerances on drawing sheets [1]. The first tool is the analysis of 1D tolerances, using worst-case analysis. The second tool is to use commercial 3D CAT (Computer Aided Tolerancing) software programs, which perform the analysis with statistical methods and the worst-case method, but based on point-to-point restrictions or perfect features, among the components. One typical example is the simulation of contact between two plane surfaces in CAT with point-to-point tools or with features, which differs from the real contact of these surfaces. Both 1D and 3D CAT programmes assist designers with their decision-making processes in relation to the values that the tolerances should take, but not with their optimization.

There is as yet no methodology for determining the Dimensional and Geometrical Tolerances (GD\&T) that are specified in drawings, which is still an unresolved problem. Concurrent engineering in design and manufacturing departments, together with the past experience of 
their staff in the design of similar mechanisms to those that the departments are designing at any one time, are of vital importance to define the GD\&T of each of the parts of a new mechanism. Previous experience is not sufficient to achieve the largest possible tolerances that comply with the Functional Requirements (FR) imposed at the design stage; tolerance analysis software is needed that simulates the behaviour of each of the tolerances that influence the tolerance chain stack up of each functional requirement. The same tolerance is often associated with various FR and therefore with various dimension chains. To understand its optimum value, so that the cost of manufacturing of the mechanism is as low as possible, requires other tools in addition to CAT software. A matrix table needs to be designed, in which all of the FR and all of the GD\&T of the mechanism may be seen, so as to confirm the effect of modifying one of these tolerances on the different functional requirements which are influenced by this GD\&T. We refer to this matrix table as the "dimensional hierarchization matrix".

In this work, our aim is not to solve a tolerance analysis problem, but to present the joint methodology of 3D CAT software with the "dimensional hierarchization matrix" and an optimization algorithm that is used in a firm, so that GD\&T may be optimized and manufacturing costs reduced.

\section{Backgrounds}

\subsection{Functional Analysis}

Functional analysis consists of identifying the requirements that have to be met between the components, so as to guarantee the assembly of the parts and the functional operation in the mechanism. It is divided into two principal phases: the functional analysis of the engineering expectations, where the performance specifications are detailed; and the technical/functional analysis that examines the assembly of the mechanism and its installation at the client's chosen location. Having analyzed functional aspects and the assembly of the mechanism, the designer should identify the functions and sub-functions that the mechanism has to perform. Each sub-function should be converted into a functional requirement such as, for example, maximum and minimum clearance or maximum and minimum distances between two points or two axes. Initially, each of the FR are identified, along with the dimensions and the tolerances in the dimension chain of that FR, thereby establishing the functional components of the different parts, but not their dimensional values or their GD\&T. The initial values of the GD\&T are normally set on the basis of the previous experience of the firm's product design and manufacturing team. CAT software simulates the GD\&T that are estimated for each part and verifies their degree of compliance with the FR of the mechanism or otherwise. The dimensions of highest and of lowest importance in the different FR are identified, by means of the percentage contribution of the different dimensions to the FR under study. The GD\&T of some parts are modified, searching for the maximum tolerances that are compatible with the different FR. This process of modification of the GD\&T and their subsequent simulation using CAT software may be repeated a number of times until optimum GD\&T for compliance with the FR are found.

It is true that some mathematical algorithms exist that search for minimum manufacturing costs in the equations of unidimensional dimension chains, thereby obtaining optimum GD\&T values, as in [2], although they are not included in 3D CAT software. These mathematical algorithms are for FR in 1D and begin with the assumption that the sensitivities are known and that the FR equations may be expressed in a linear way. However, the linear models of the FR have limitations in 3D [3]; when special or complex geometrical tolerances are specified, such as Maximum Material Condition (MMC), and compound tolerances; when 
non-normal distributions are specified; and in overly-constrained systems. Governi [4] used a genetic algorithm in CAT software called eTol-Mate to optimize costs, which sets certain cost coefficients that are normally unknown in the design. It is therefore very important for the designer to be able to visualize the key GD\&T, at a glance, using both CAD and CAT. There is no need to make direct use of cost algorithms, but rather to use them indirectly through process capabilities. We propose to design the "dimensional hierarchization matrix" so that it may be displayed on an Excel spread sheet. Through its different iterations, this matrix allows us to see which of the tolerances should first be modified to optimize the design requirement specifications.

\subsection{Theoretical Framework}

Tolerance analysis in design begins with an analytical model that examines the tolerance chains in a mechanical assembly of component parts. The three most common models used in engineering design are: 1- The Worst-Case (WC), 2- The Root Sum Squares (RSS), 3- The Monte Carlo method. The RSS and Monte Carlo methods are statistical methods, and the WC method determines the maximum absolute variation of an FR by accumulation of tolerances.

The relation, $f$, between the input variables, $x_{i}$, in the tolerance analysis of a functional requirement $(y)$, determined by the variables $\left(x_{i}\right)$ in their dimensional chain, $y=f\left(x_{1}, x_{2} \ldots x_{i}, \ldots x_{n}\right)$, may in certain cases be an unknown value. If so, a Monte Carlo simulation is needed with a $3 \mathrm{D}$ CAT package from the 3D CAD model. However, there are many other cases, in tolerance analysis, where the relation, $f$, between the input variables, $x_{i}$, is linear, and may be expressed with an approximation of a first-order Taylor equation: $y=\sum_{i=1}^{n}\left|\frac{\partial f}{\partial x_{i}}\right| \cdot x_{i}{ }^{\prime}$ where $\frac{\partial f}{\partial x_{i}}$ is the first partial derivate of the function and is referred to as its sensitivity.

Description of the three methods:

1- Worst Case Method (WC). In a worst limits analysis, the assembly tolerance (Ty) is determined by a linear sum of the component tolerances $\left(T x_{i}\right)$. Each component dimension is assumed to be at its maximum or minimum limit, yielding the worst possible assembly limits $[5]$.

- If the dimension chain is in $2 \mathrm{D} / 3 \mathrm{D}$, the tolerance of the functional condition of the assembly is: $T y=\sum_{i=1}^{n}\left|\frac{\partial f}{\partial x_{i}}\right| T x_{i}$

This formula is obtained as the difference between the extreme values of $y, T y=y_{\max }-y_{\min }$, and $T x_{i}$ is also obtained as the difference between the extreme values of $x_{i} \quad T x_{i}=x_{i_{\max }}-x_{i \min }$

The tolerance contribution is the weight of one of the tolerances of the dimension chain in the tolerance of the assembly, such as:

Contribution_ $x_{j}=\frac{\left|\frac{\partial f}{\partial x_{j}}\right| \cdot T x_{j}}{\sum_{i=1}^{n}\left|\frac{\partial f}{\partial x_{i}}\right| \cdot T x_{i}} \cdot 100$

The definition of the percentile contribution of each tolerance is important, because it helps identify the tolerances that we should modify for optimal assembly conditions. 
- If the dimension chain is in 1D and the sensitivities are one, $T y$ is: $T y=\sum_{i=1}^{n} T x_{i}$

2- RSS Method. The RSS method is used with 3 dimensions in the FR. The low probability of the WC combination occurring is statistically accounted for, by assuming a normal distribution of the component variations. The RSS method is based on the following error transmission formula: $\sigma_{y}^{2} \approx\left(\sum_{i=1}^{n}\left|\frac{\partial f}{\partial x_{i}}\right|\right)^{2} \cdot \sigma_{i}^{2}$. Its solution yields the two formulae that appear below, assuming that the tolerances of an assembly, $T y$, are 6 times the standard deviation $\left(\sigma_{y}\right)$ and that the tolerance of a dimension of a single component $\left(T x_{i}\right)$ is 6 times $\left(\sigma_{i}\right)$.

- If the dimension chain is in $2 \mathrm{D} / 3 \mathrm{D}$, assuming the independence of the variables:

$T y=\sqrt{\sum_{i=1}^{n}\left(\frac{\partial f}{\partial x_{i}}\right)^{2} \cdot T x_{i}^{2}}$

Contribution_ $x_{j}=\frac{\left(\frac{\partial f}{\partial x_{j}} \cdot T x_{j}\right)^{2}}{\sum_{i=1}^{n}\left(\frac{\partial f}{\partial x_{i}} \cdot T x_{i}\right)^{2}} \cdot 100$

- If the dimension chain is in $1 \mathrm{D}, T y=\sqrt{\sum_{i=1}^{n} T x_{i}^{2}}$ with sensitivities equal to 1 .

If it is assumed that the distributions of the variables are uniform for $1 \mathrm{D}$, then $T y=\sqrt{3} \cdot \sqrt{\sum_{i=1}^{n} T x_{i}^{2}}$

However, if the FR may not be expressed in a linear form and/or the distributions are not normal, then the Monte Carlo method should be used.

3- The Monte Carlo method. The Monte Carlo method estimates the dimensional variation in an assembly, due to the dimensional and the geometric variations of the different components in the assembly. This iteration process estimates the mean, the standard deviation, the coefficients of asymmetry and kurtosis and the percentile rejection of the output variable (Fig. 1).

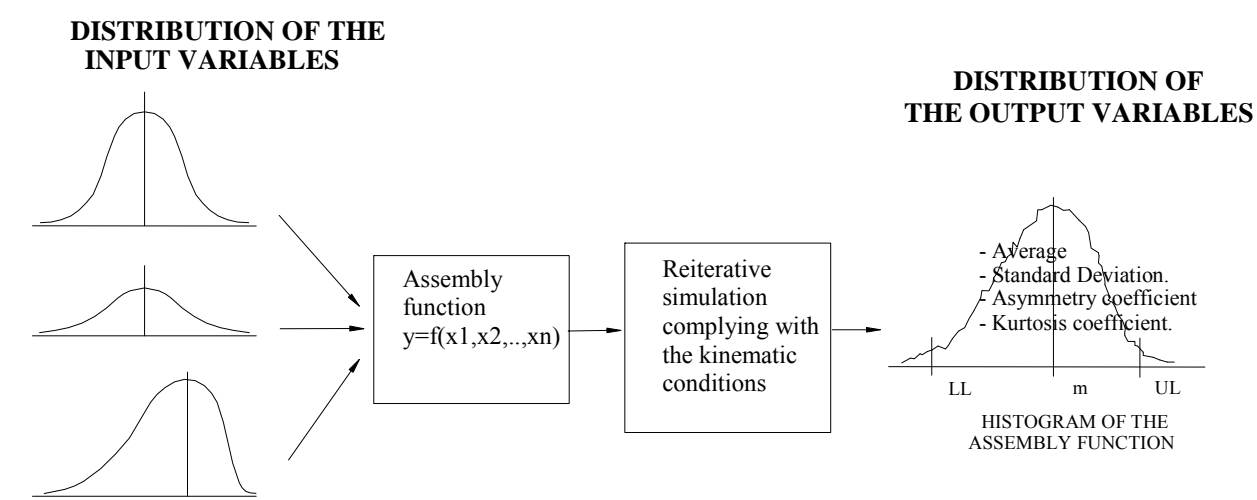

Fig. 1. Monte Carlo method

Whether the distribution of the input variables are known or estimated, we can statistically estimate the output variable and its distribution in the assembly, provided that the assembly function is known or may be simulated in the CAT.

The simulation consists in selecting random variables of the entrance variables as a function of each of its statistical distributions and calculating the resultant dimension of the assembly function. The process is successively repeated a high number of times by iteration. 
According to Gao [6] and Cvetko [7], the number of iterations is very important. A total of 1000 iterations is sufficient to determine the means and the variations of the output variable, but it is neither sufficient to determine the third and fourth-order moments (symmetry and kurtosis), nor the rejection percentage, where at least 10000 iterations should be made. Values of over 3000 iterations yield reasonably precise results.

The GD\&T values in the dimension chains are assigned by designers on a trial and error basis, in the light of their previous experience (in design, manufacturing systems capabilities and assembly tolerances) and the tolerance stack up of the standard parts. Initially, a set of values are assigned to the tolerances of the input dimensions in the dimension chains, to test whether they comply with the FR, assuming that those requirements follow normal or uniform distributions, as the statistical distributions of their manufacturing process are unknowns. If some of the FR are not met, the designers need to determine which and how many tolerances to modify. There are two parameters available to establish the tolerances that need to be modified: sensitivity and percentile contribution [1]. However, the process capability indices in the assemblies $\left(c_{p}\right.$ and $\left.c_{p k}\right)$ are the real source of the information on which of the tolerances to modify, where the $\mathrm{c}_{\mathrm{pk}}$ index reflects the eccentricity of the process with regard to the estimated mean $\mu$ of the process. These indices are:

$c_{p}=\frac{U S L-L S L}{6 \sigma} \quad c_{p k}=\min \left(\frac{U S L-\mu}{3 \sigma}, \frac{\mu-L S L}{3 \sigma}\right)$, where $U S L$ and $L S L$ are the upper and the lower limits specified in each of the FR and $\sigma$ is the standard deviation of the FR in the simulation of the process [8].

3D CAT software programmes allow us to confirm whether the functional tolerances obtained in the simulation are larger or smaller than the tolerances specified in the FR. They also allow us to identify and to calculate the eccentricity values that will subsequently be corrected, by modifying the dimensional limit values. However, they are of little use for the optimal calculation of the GD\&T of the input variables. It is therefore necessary to use a methodology that will allow us to modify the initial values of the GD\&T, by approximating them to optimal values, taking into account the capabilities of the different manufacturing processes. The use of a matrix table or double-entry spread sheet showing the FR and with the GD\&T, in which the contributions of the GD\&T are indicated and their compliance with the FR values, would serve to identify, in a methodological way, the GD\&T that should be modified. As mentioned, we refer to the table as the "Dimensional hierarchization matrix".

\subsection{Tolerance Analysis Software}

The principal 3D CAT tolerance analysis software programmes are: Tecnomatix Variation Analysis (VSA), Tecnomatix eM-TolMate, Enventive, 3-DCS, CETOL and Sigmund. These packages conduct a tolerance analysis using either the linear equations method or the Monte Carlo method. Commonly available results are the lists of contributors, sensitivities, \% contributions (to variance), and tolerance accumulation for worst-case scenarios and statistical analysis. In general, these CAT software packages import the 3D geometry of each part in IGES or STEP format, but in no case import their GD\&T, which have to be fed into the CAT by means of feature abstraction. For example, a plane in the CAT may be defined by means of three points in the 3D model, which may be moved, following a statistical distribution, in a perpendicular direction to the aforementioned plane. The choice of these three points of the plane and the type of statistical distribution of its movements is done by the analysts and designers on the basis of their previous experience. Commercial CATs basically use pointbased analysis that fails to reflect true 3D tolerance zones and geometric variations.

There are three main types of variation in an assembly: 
- Variations due to (longitudinal and angular) dimensional tolerances.

- Variations due to geometric tolerances (position, perpendicularity, roundness, flatness...).

- Kinematic variations (small displacements in the joints between the parts).

The first two are the result of variations in the GD\&T of the individual components in the manufacturing processes and should be adjusted to the standards $[9,10]$ and the third variation is due to the assembly process of the final product. $\mathrm{Lu}$ [11] demonstrated that different assembly sequences can produce important variations in the simulation of the results in the FR. Ramos [11] demonstrated that the axis eccentricity of a rivet pin position in a hole with clearance was approximately half of the maximum clearance.

Polini [13] compared the following tolerance analysis methods: Vector loop, Variational, Matrix, Jacobian and Torsor; and came to the conclusion that the vector loop model and the variational model appeared to be more developed than the others; these models were the only ones that provided support for solving tolerance stack-up functions involving networks. Moreover, they provided a method for assigning probability density functions to the model parameters, given the applied tolerances. However, the vector loop model and the model with variations were not completely consistent with the actual ISO and ASME standards and failed to provide support for handling interactions between tolerance zones. We employed Sigmund CAT software that uses the variation tolerances analysis method.

\subsection{Dimensional hierarchization matrix}

One type of matrix that relates the FR and the GD\&T was established by Islam [14], who placed the FR in the rows and the GD\&T in the columns, so as to indicate with an ' $x$ ' which of the GD\&T were related to the different FR. It could thereby be confirmed whether a GD\&T influenced one or various FR. Islam [14] began with the application of 1D dimension chains to four specific cases (adjustments, longitudinal dimensions, a combination of both and surface textures), where the FR were mathematically defined in an explicit way, but took no account of the Geometric Tolerances (GTs).

The incorporation of 3D CAT in many cases implies no explicit mathematical knowledge of the FR equation, as the sensitivity of some of the input variables are unknown. But the CAT simulation allows us to ascertain the contribution of each (geometric and dimensional) tolerance for each FR. If we know the Upper Specification Limit (USL) and the Lower Specification Limit (LSL) of the FR included in the design and if we carry out a CAT simulation of the GD\&T in the drawing, we can obtain the values of the FR in a random way using Monte Carlo simulation in the CAT. Obtaining a frequency distribution and the Upper Control Limit (UCL), Lower Control Limit (LCL) and mean values of the FR simulation, the process capability indices $\left(\mathrm{c}_{\mathrm{p}}\right.$ and $\mathrm{c}_{\mathrm{pk}}$ ) may therefore be found and the \% values of each of the FR that are outside of the design values ("\% Out"). These values serve to identify the GD\&T that should be modified and the manufacturing processes with which these GD\&T comply.

The dimensional hierarchization matrix in table 1 is therefore proposed, which contains the following data:

- The FR (FR-1 .........FR-n) are listed horizontally.

- Each part of the mechanism is listed vertically with its initially proposed dimensions and GD\&T, which influence each FR (d1.1, t1.1....).

- A column with the clearance values or the USL and the LSL of the FR.

- Columns with the UCL, "Mean or $\mu$ " and LCL of the GD\&T simulation, obtained in the CAT. 
- Columns with the values: process capabilities indices $\left(c_{p}, c_{p k}\right), \%$ values of the FR outside the design values ("\% Out"), and the eccentricity between the mean values of the simulation process ("Mean") subtracted from the mean value between the lower (LSL) and the upper specification limits (USL) of the FR, which we will refer to as the 'Mean shift'. These values were also obtained in the CAT

- The manufacturing processes of each part are listed in the last row. The minimum tolerance values that may be obtained for each dimension, geometric element and feature, in each part, which are a function of their manufacturing process are shown in the penultimate row. The proposed tolerance values, $\mathrm{t} 1.1, \mathrm{t} 1.2 \ldots$ should never be lower than the minimum tolerances of their manufacturing process, which are noted in the penultimate row. Swift [14] indicated the minimum tolerance values that may be obtained in the different manufacturing processes, which may also be noted in this penultimate row.

Table 1. Dimensional hierarchization matrix

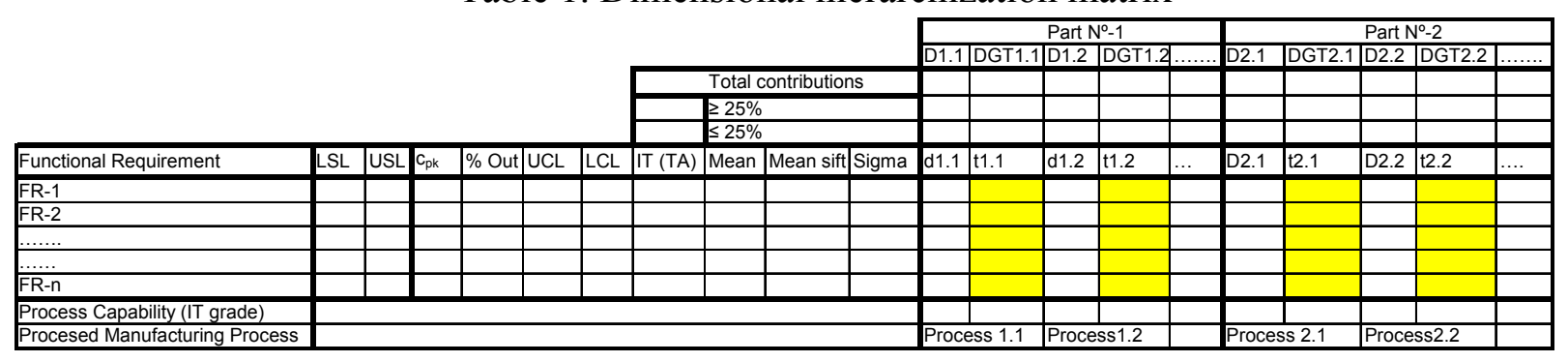

The initial values of the proposed GD\&T may be consulted in the following standards: ISO 2768-1 [16], ISO 2768-2 [17], and ISO 8062-3 [18], which indicate both the dimensional and the geometric general tolerances according to whether the quality is high, medium or low; and/or in accordance with the past experience of the concurrent engineering team. Once the initial values of the GD\&T and the values of the FR are known, the GD\&T of each part of the mechanism are simulated in the CAT using Monte Carlo, to obtain (Fig.9):

- The contributions of each GD\&T, the number of which are higher and lower than $25 \%$, and the FR that they affect.

- The UCL, mean and LCL values obtained in the CAT simulation of each FR. Therefore, the interval simulated in the CAT of the tolerance associated with each of the FR in the assembly (TA); TA=UCL-LCL.

- The percentage values of the FR outside the specifications "\% Out", the process capabilities $c_{p}$ and $c_{p k}$ and the 'Mean Shift' eccentricity of the FR.

Finally, the hierarchization matrix is completed with the values obtained in the CAT (table 3 ). The values of this table are copied and pasted in the Excel algorithm, with the aim of optimizing the tolerances.

\section{Tolerance optimization}

The tolerance optimization process may be split into two parts:

a) Centring of the process.

The average eccentricity value is: Mean_Shift $=\frac{U S L+L S L}{2}-$ Mean, which has to be corrected by increasing the nominal values of the dimensions in the dimension chain (if the eccentricity is positive), or by reducing the nominal values (if negative). The correction value may be completed in a single dimension or may be shared among all of the dimensions in the dimension chain. This centring means that each $\mathrm{c}_{\mathrm{pk}}$ value approximates to the $\mathrm{c}_{\mathrm{p}}$. 
b) Optimization tolerances $\mathrm{Tx}_{\mathrm{i}}$.

It may for example be the case that acceptable tolerance stacks give process capability $\left(\mathrm{c}_{\mathrm{pk}}\right)$ values of between 1.3 and 1.5. Therefore, when the $c_{p k}$ values in certain FR are below 1.3, some of the GD\&T related to those FR should be reduced; and when the $\mathrm{c}_{\mathrm{pk}}$ values are higher than 1.5, an attempt should be made to increase the GD\&T. On many occasions, a tolerance may be reduced in an FR, because its $\mathrm{c}_{\mathrm{pk}}<1.3$, which implies that this tolerance may not be increased in another FR. The new values of the proposed GD\&T will be higher than the minimum tolerances of the manufacturing processes $\left(\mathrm{Tx}_{\mathrm{i}}\right)_{\min }$ that were initially proposed. Were it not possible to reduce the GD\&T further, a change of manufacturing process would be necessary.

The hierarchization matrix allows us to see, at a glance, which of the GD\&T may be reduced or increased in accordance with their $\mathrm{c}_{\mathrm{pk}}<1.3$ or $\mathrm{c}_{\mathrm{pk}}>1.5$, but it gives no information of by how much. In the first place, those GD\&T with contributions of over $25 \%$ are reduced, if their $\mathrm{c}_{\mathrm{pk}}<1.3$. Subsequently, those GD\&T with contributions below $25 \%$ are increased if their $\mathrm{c}_{\mathrm{pk}}$ $>1.5$ and no FR influenced by this GD\&T has a $c_{p k}<1.3$. The new values of the $\operatorname{Tx}_{\mathrm{i}}$ tolerances may be adjusted by trial and error or by taking account of the limit value of the new $\mathrm{Tx}_{\mathrm{i}}$ $\left(T x_{i}=\frac{c_{p k_{j}}}{1,3} T x_{i}\right)$, although their influence on the hierarchization matrix must be observed at all times. This iterative process of modification of the GD\&T and simulation in CAT may be repeated until the concurrent engineering team considers that the GD\&T values comply with the FR.

A second tolerance optimization method is to use an algorithm in Excel, which follows the aforementioned rules. Four algorithms were assessed, before the flow diagram in figure 2 was selected.

This algorithm starts by centring the process, increasing or decreasing the mean dimensional values or the nominal dimensions until the Mean Shift $t_{j}$ is corrected. Following the centring, the $\left(c_{p k}\right)_{j}$ of all the FR and the new $\mathrm{Tx}_{\mathrm{i}}$ are obtained from the previous $\mathrm{Tx}_{\mathrm{i}}$, in the FR to which those tolerances apply, by means of the following formula: $T x_{i}=\frac{c_{p k_{j}}}{c_{p k_{\min }}} T x_{i}$

Each new $\mathrm{Tx}_{\mathrm{i}}$ value is chosen from among those obtained from the different $\mathrm{FR}$ that affect that $\mathrm{Tx}_{\mathrm{i}}$, taking into account the following conditions and their order of priority:

1- At no time can $\mathrm{Tx}_{\mathrm{i}}<\mathrm{Tx}_{\mathrm{i} \text { min }}$, the minimum manufacturing process tolerance (D). If otherwise, $\mathrm{Tx}_{\mathrm{i}}$ should be increased or a process with lower minimum tolerances should be introduced.

2- If some $\mathrm{c}_{\mathrm{pk}}<1.3$ in some $\mathrm{Tx}_{\mathrm{i}}$, then $\mathrm{Tx}_{\mathrm{i}}$ must be reduced, selecting the lowest $\mathrm{Tx}_{\mathrm{i}}$ from all of the rows that have $\mathrm{c}_{\mathrm{pk}}<1.3(\mathrm{~B})$

3- If some $1.3 \leq \mathrm{c}_{\mathrm{pk}} \leq 1.5$, there is no need to change the $\mathrm{Tx}_{\mathrm{i}}$ which affect these $\mathrm{c}_{\mathrm{pk}}(\mathrm{A})$.

4- If the tolerance $\mathrm{Tx}_{\mathrm{i}}$ has an influence on some $\mathrm{c}_{\mathrm{pk}}>1.5$ and is not included in any of the three earlier cases, $\mathrm{Tx}_{\mathrm{i}}$ increases, selecting the lowest $\mathrm{Tx}_{\mathrm{i}}$ from the rows that have a $\mathrm{c}_{\mathrm{pk}}>1.5(\mathrm{C})$.

The order with which the value of each $\mathrm{Tx}_{\mathrm{i}}$ is chosen, taking account of the FR that affects it is as follows: $1^{\circ}-\mathrm{D} ; 2^{\circ} \mathrm{B} ; 3^{\circ} \mathrm{A}$ and $4^{\circ} \mathrm{C}$.

If after the four steps, some $\mathrm{c}_{\mathrm{pk}}<1.3$, this is corrected in the following iteration by reducing the $\mathrm{Tx}_{\mathrm{i}}$, as in the second step. 


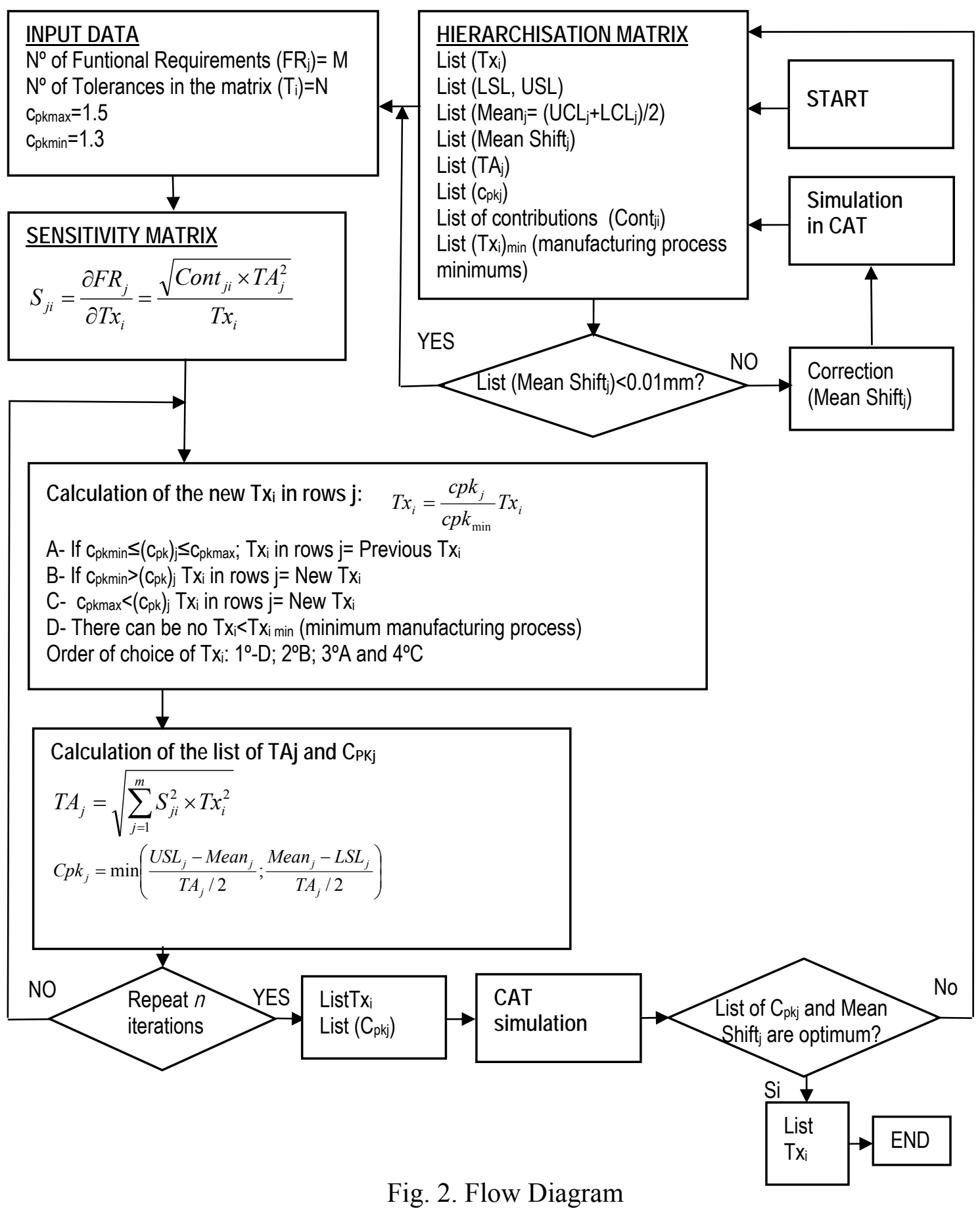

\section{Results of the tolerance analysis methodology}

A functional and tolerance analysis of an automobile window winder mechanism manufactured at Grupo Antolín (an international firm in the automobile components sector) was performed to test the advantages of the combined methodology of 3D CAT, the dimensional hierarchization table and the optimization algorithm.

We focused on the functional condition that the pulley and the rivet pin have to be fixed to the rear guide rail of the window, so that the cable will not slip off the pulley and at the same time so that it can be assembled: in particular, the FR of the clearance between the pulley and the rail, which we refer to as $(\mathrm{J})$. See figures 3,4 and 5 . 

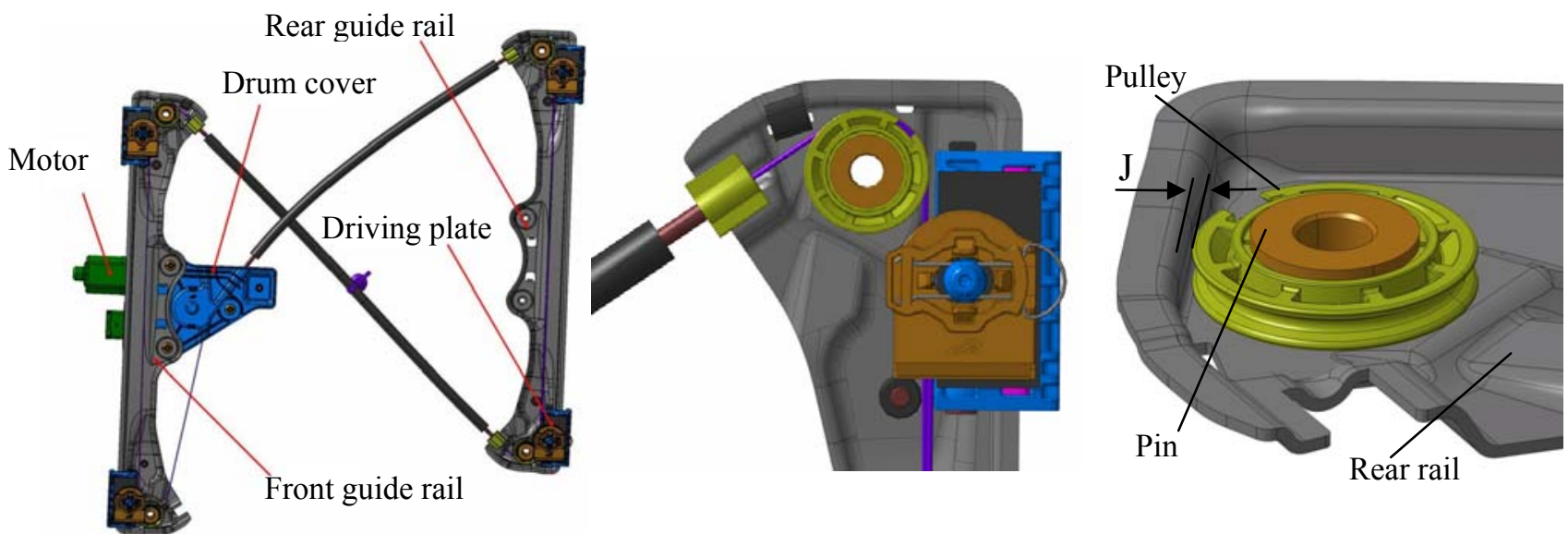

Fig. 3. Window regulator

Fig. 4. Detail of window regulator. Fig. 5. Functional Requirement (J)

Prior to this study, the analysis of the dimension chains in the FRs was done by using the spread sheet in table 2. It represents a sketch of the functional condition ' $J$ '. The average value and the tolerance of ' $\mathrm{J}$ ' was obtained by three different tolerance analysis methods in 1D: arithmetic (WC), quadratic (RSS) and probabilistic (uniform distribution). Ngoi [19] and Shen [20] used this method, but only to analyze tolerances in the WC method.

Only the 2 critical positions may be taken into account in Excel that are shown in figure 6 . The first defines the minimum distance $(\mathrm{Jm})$ between the pulley and the rail, the contact points occurring at A. In the second, the maximum distance $(\mathrm{JM})$ was defined, the contact points occurring at B.
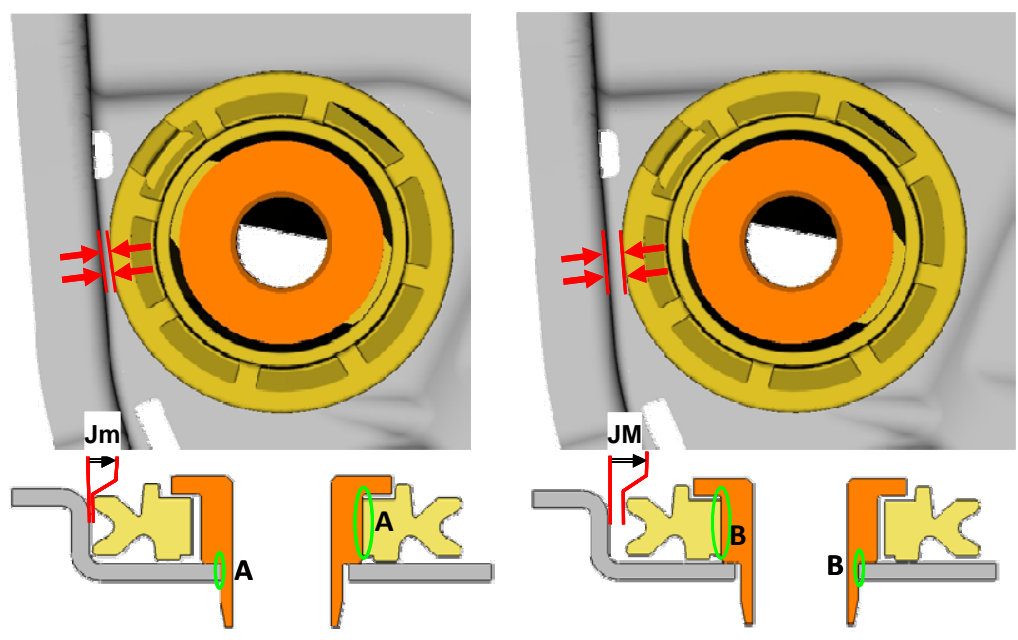

Fig.6. Extreme positions of the clearance

In table 2, it may be seen that, if we consider the two positions and the worst cases, the clearance values between the pulley and rail would vary between 1.55 and $0.05 \mathrm{~mm}$. 
Table 2. 1D tolerance analysis on an Excel spread sheet

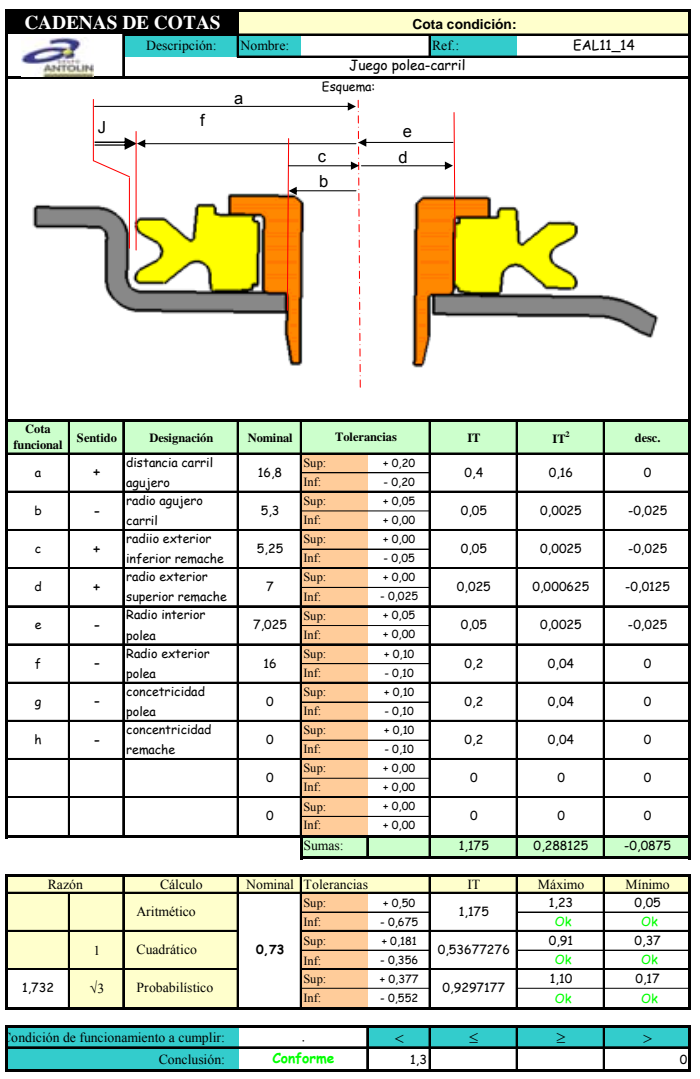

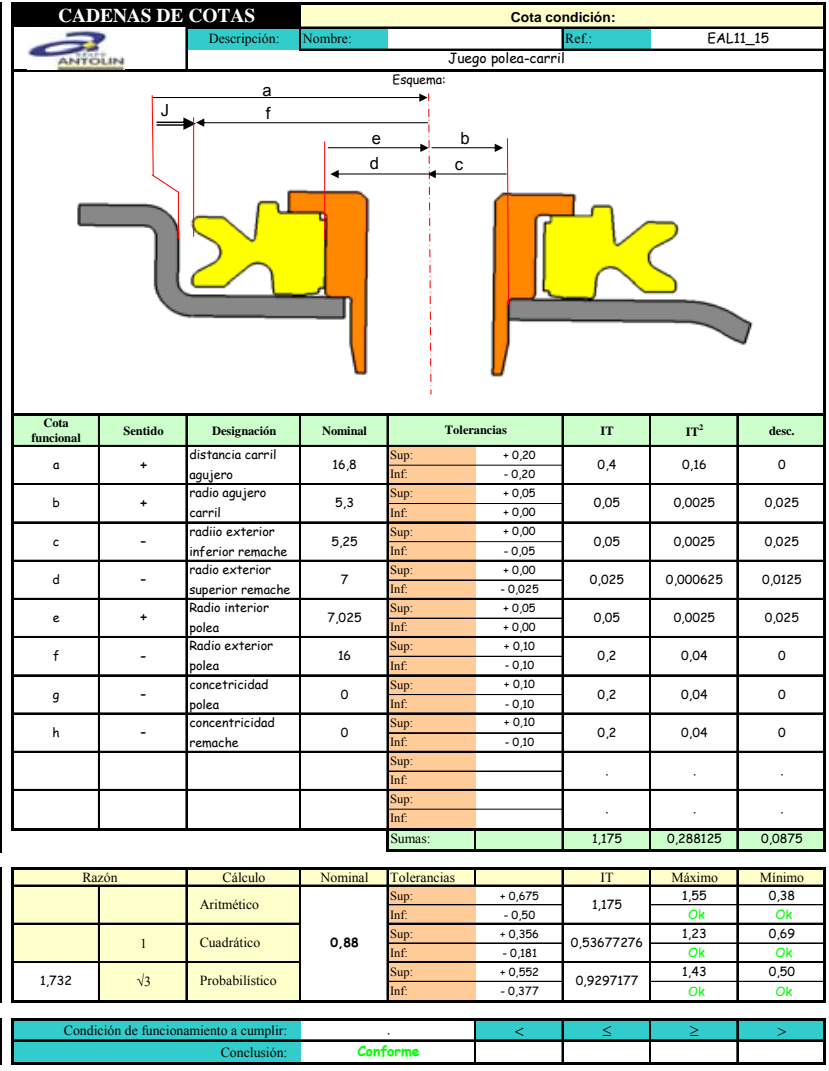

\subsection{Simulation in CAT.}

At present, 3D CAT software programmes such as Sigmund are used to complete the tolerance analysis in the assembly. Sigmund software completes the simulation in various steps:

1. Component assemblies: where it is necessary to define the reference systems at each component so that they may then be assembled (Fig. 7). These reference systems will be used to indicate the extent to which the parts will move in the slight displacements of the couplings between an axis and a hole, which is known as "hole-pin float".
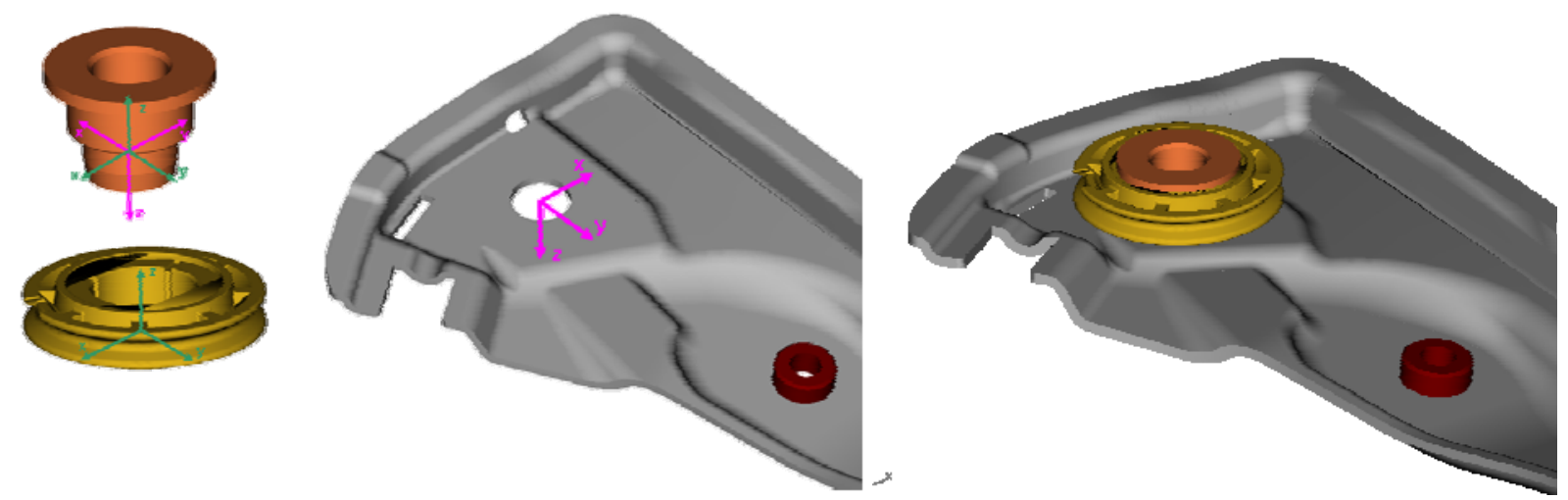

Fig. 7. Pulley assembly and rivet in the rail

2. Definition of the deviations of the parts: firstly, by indicating the way in which the "holepin float" joints between the rail and the rivet pin and between the rivet pin and the pulley are assembled. At which point, the dimensions and tolerances of the axis and the hole, together with the radial and the angular variations of the joint are introduced (Fig. 8). 
3. Definition of the FR to study. In this example J (see Fig. 5).

4. Monte Carlo simulation of the model with 10,000 samples.

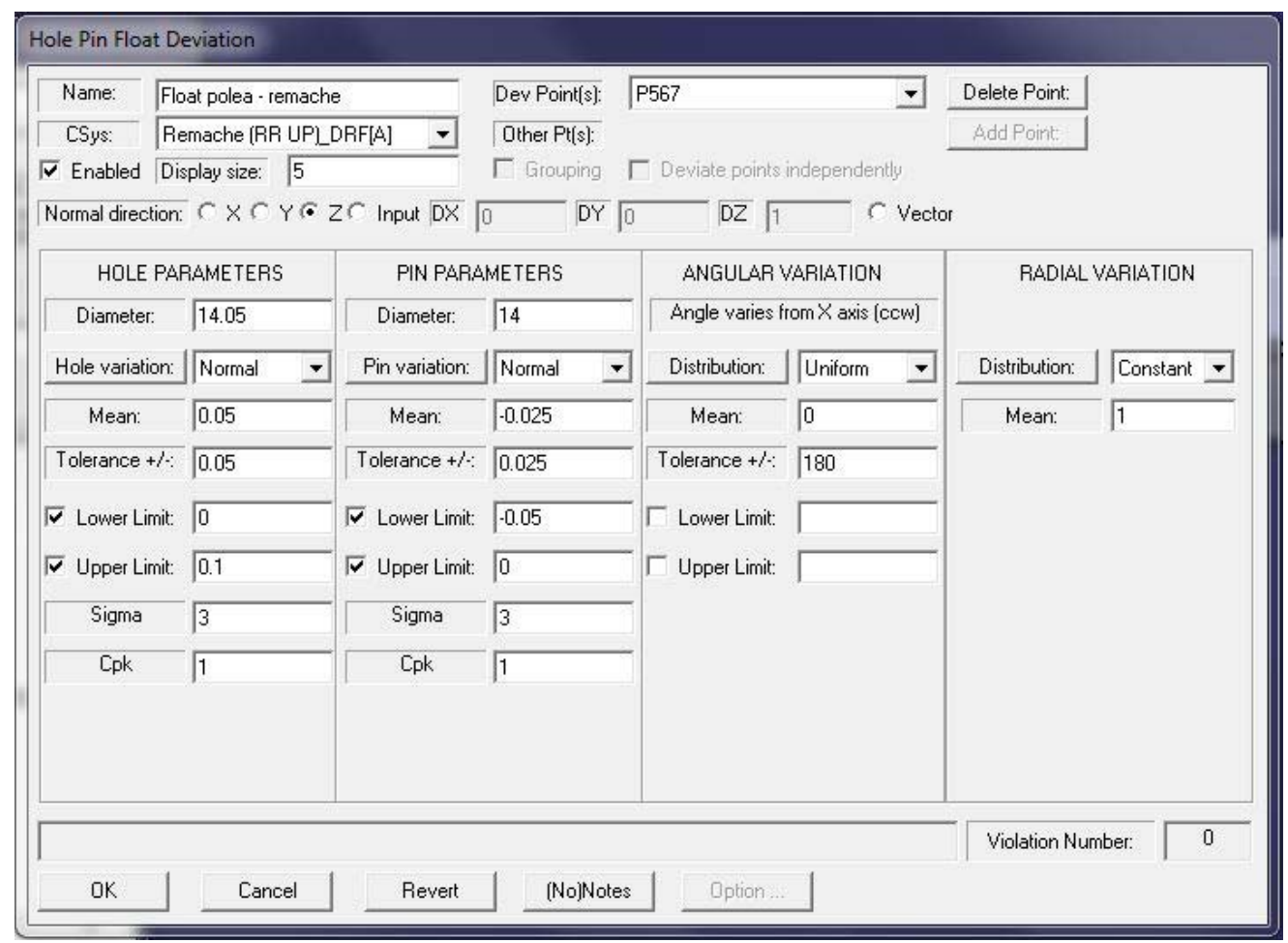

Fig.8. Definition of hole-pin float

5. Verification of the results. Sigmund generates the report on all the FR, in the example of FR ' $J$ ', in figure 9, where we are given information on the contributions of each GD\&T that influences the FR, and on the LCL, Mean, UCL, $\mathrm{c}_{\mathrm{p}}, \mathrm{c}_{\mathrm{pk}}$, and \%Out...

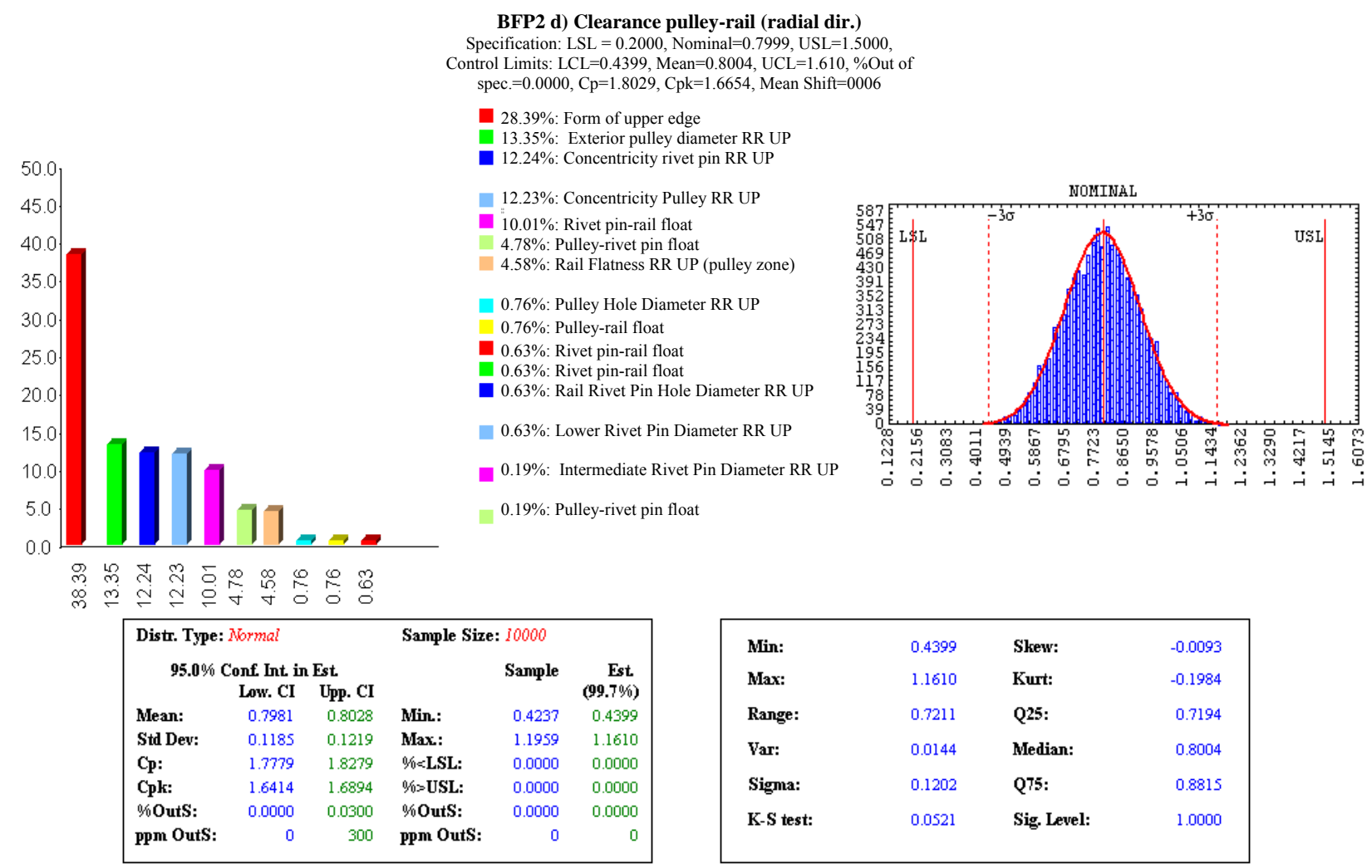

Fig. 9. Sigmund Report on FR 'J' 
Having obtained all of the Sigmund reports on all the FR, these are inputted into the dimensional hierarchization matrix, so as to visualize in an easy and simply way which of the FR may be modified to optimize the tolerances of the window regulator. A small part of the complete dimensional hierarchization matrix is shown in table 3.

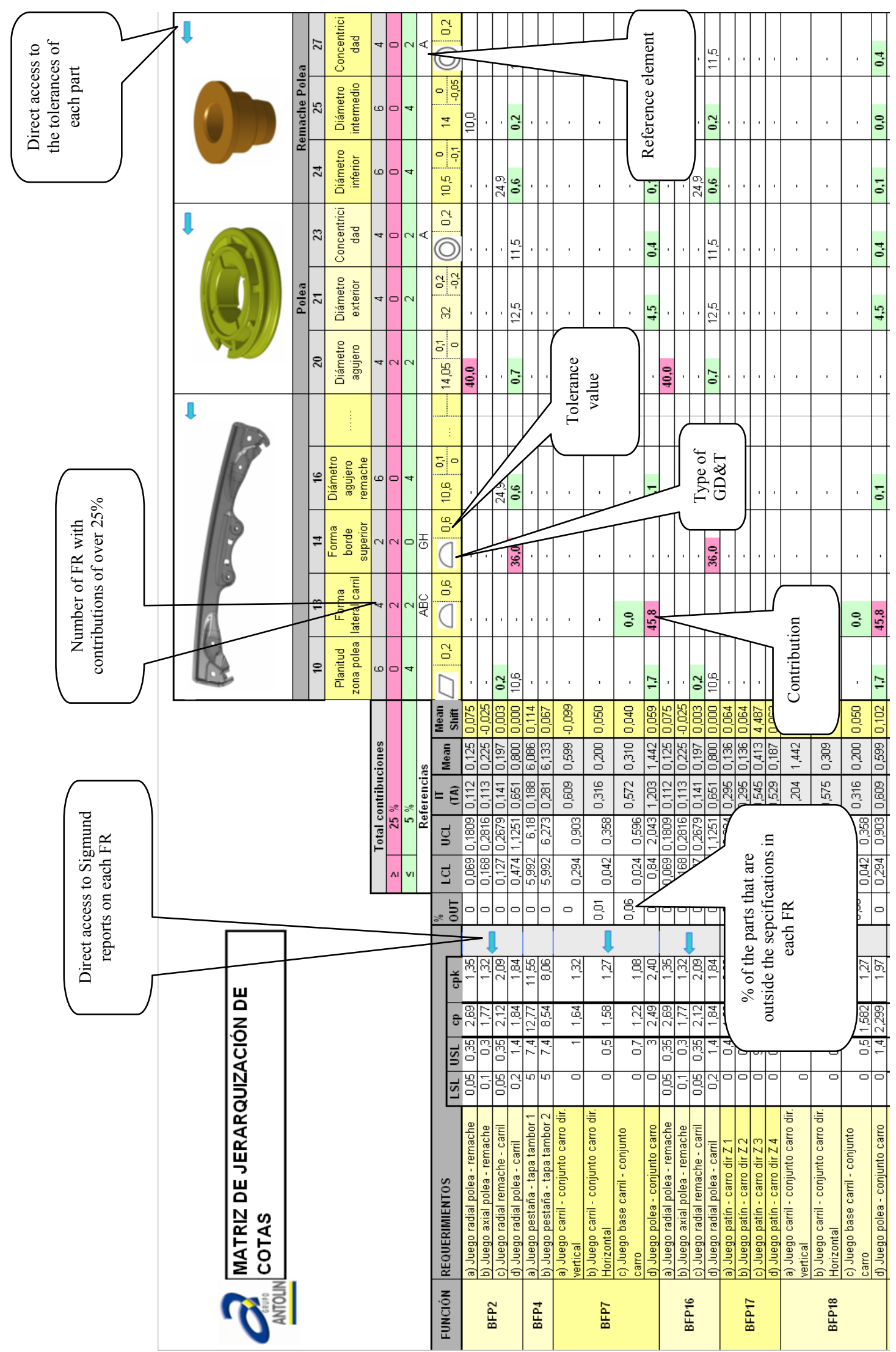


Which shows:

- The GD\&T and their percentage contribution to each of the FR.

- The FR that are off-centre.

- A report on the dimensions, tolerances and graphics may be accessed by clicking on the blue arrows in the drawing of each part. The Sigmund reports on each FR may be accessed by clicking on the blue arrows in the column.

- The total number of FR that each GD\&T influences can be seen in a row. It also records the number of FR that contribute above $25 \%$ and below $5 \%$ in each GD\&T, with a view to the rapid identification of which tolerances we should adjust, either to reduce costs or in the case of non-compliance, to comply with the FR.

- The column "\%Out" shows the $\%$ of the parts that fall outside of the specifications in each FR.

In this way, the nominal dimensions that should be centred and the GD\&T that should be modified are identified and they are modified by means of a $\mathrm{Tx}_{\mathrm{i}}$ optimization algorithm; and once again, the simulation process in Sigmund is repeated. The whole process is repeated until the concurrent engineering team considers that the new tolerance values are valid.

If we compare the results of the earlier method of tolerance analysis (1D Excel spread sheet) and the present method (3D Sigmund), we can see that the results differ substantially; the closest results to those from sigmund were provided by $1 \mathrm{D}$ Excel for the quadratic/probabilistic solution (see Table 4).

Table 4. Comparison 1D-3D

\begin{tabular}{|l|c|c|}
\hline \multicolumn{1}{|c|}{ Calculation } & Maximum & Minimum \\
\hline Arithmetic 1D & 1.55 & 0.05 \\
\hline Quadratic 1D & 1.23 & 0.37 \\
\hline Probabilistic 1D & 1.44 & 0.17 \\
\hline Sigmund 3D & 1.13 & 0.47 \\
\hline
\end{tabular}

1D tolerance analysis in assemblies using the Excel sheet (Table 2) fails to calculate the contributions, because it does not take into account the sensitivities, but if we look at the graph of that table, we can affirm that the different sensitivities are 1 and we can thereby obtain the contributions of the analysis of arithmetic and quadratic tolerances in table 2 . The contributions calculated by Sigmund are shown in figure 9.

Table 5. Comparison of contributions

\begin{tabular}{|c|l|c|c|c|l|}
\hline $\begin{array}{c}\text { Functional } \\
\text { dimension }\end{array}$ & Designation in 1D Excel & $\begin{array}{c}\text { Arithmetical } \\
\text { Contribution }\end{array}$ & $\begin{array}{c}\text { Quadratic/ } \\
\text { probabilistic } \\
\text { contribution }\end{array}$ & $\begin{array}{c}\text { Contribution } \\
\text { of Sigmund }\end{array}$ & Designation in Sigmund \\
\hline $\mathrm{a}$ & Distance rail - hole & $34.04 \%$ & $55.53 \%$ & $38.39 \%$ & Rail edge position \\
\hline $\mathrm{b}$ & Radius hole - rail & $4.26 \%$ & $0.87 \%$ & $1.26 \%$ & Rail hole diameter \\
\hline $\mathrm{c}$ & Lower exterior radius of rivet pin & $4.26 \%$ & $0.87 \%$ & $1.26 \%$ & Lower rivet pin diameter \\
\hline $\mathrm{d}$ & Upper exterior radius of rivet pin & $2.13 \%$ & $0.22 \%$ & $0.38 \%$ & Upper rivet pin diameter \\
\hline $\mathrm{e}$ & Interior radius of pulley & $4.26 \%$ & $0.87 \%$ & $1.52 \%$ & Interior diameter of pulley \\
\hline $\mathrm{f}$ & Exterior radius of pulley & $17.02 \%$ & $13.88 \%$ & $13.35 \%$ & Exterior diameter of pulley \\
\hline $\mathrm{g}$ & Concentricity of pulley & $17.02 \%$ & $13.88 \%$ & $12.23 \%$ & Concentricity of pulley \\
\hline $\mathrm{h}$ & Concentricity of rivet pin & $17.02 \%$ & $13.88 \%$ & $12.24 \%$ & Concentricity of rivet pin \\
\hline & & & & $4.58 \%$ & Rail flatness around pulley \\
\hline & & & & $10.01 \%$ & Float rivet-rail \\
\hline & & & & $4.78 \%$ & Float rivet pulley \\
\hline & & $100.00 \%$ & $100.00 \%$ & $100.00 \%$ & \multicolumn{1}{|l}{} \\
\cline { 5 - 6 } & & & & &
\end{tabular}


If we compare the contributions of these methods (table 5), we see that the tolerances with the highest contributions are the same in all four methods. We may also see that there are important differences, as the use of Sigmund means that other contributions may be quantified such as:

- Rail flatness with a contribution of $4.6 \%$.

- The hole-pin float of the pin in the rail with a contribution of $10 \%$ and the hole-pin float of the rivet pin in the pulley with a contribution of $4.8 \%$.

These new contributions are important and were not taken into account in the 1D Excel tolerance analysis.

\subsection{Centring of the dimensions}

In the first simulation in Sigmund, simulating with the tolerances of the drawing, the eccentricities that have to be corrected are obtained (Table 6). If the nominal or mean dimensions of the pieces that correct these eccentricity values are modified (diameter of the pulley hole $+0.075 \mathrm{~mm}$, height of the intermediate cylinder of the rivet pin $-0.025 \mathrm{~mm}$ and diameter of the rail hole $+0.003 \mathrm{~mm}$ ) and the simulation is repeated in Sigmund, we obtain new values of $c_{\mathrm{pk}}$, which in general are greater and that now coincide with their $\mathrm{c}_{\mathrm{p}}$. This will allow us to use higher values of $\mathrm{Tx}_{\mathrm{i}}$ in the optimization.

Table 6. Comparison of the $\mathrm{c}_{\mathrm{pk}}$

\begin{tabular}{|l|c|c|c|c|c|c|}
\cline { 2 - 8 } \multicolumn{1}{c|}{} & \multicolumn{3}{c|}{ Initial values } & \multicolumn{3}{c|}{ Values after centring } \\
\hline Functional Requirement & $\mathrm{c}_{\mathrm{p}}$ & $\mathrm{c}_{\mathrm{pk}}$ & Mean shift & $\mathrm{c}_{\mathrm{p}}$ & $\mathrm{c}_{\mathrm{pk}}$ & Mean shift \\
\hline Pulley-pin radial clearance & 2.69 & 1.35 & 0.0749 & 2.65 & 2.65 & -0.0002 \\
\hline Pulley-pin axial clearance & 1.77 & 1.32 & -0.0250 & 1.77 & 1.76 & $5 \mathrm{E}-05$ \\
\hline Pin-rail radial clearance & 2.12 & 2.09 & 0.0027 & 2.09 & 2.09 & $-5 \mathrm{E}-05$ \\
\hline Pulley-rail radial clearance & 1.84 & 1.84 & 0.0004 & 1.64 & 1.61 & -0.00925 \\
\hline
\end{tabular}

\subsection{Optimization of the $T x_{i}$}

After centring the dimensions, the new $\mathrm{Tx}_{\mathrm{i}}$ are obtained by using the flow diagram from figure 2 and the 3D CAT simulation is repeated, to test whether the $\mathrm{c}_{\mathrm{pk}}$ values of all the FR are equal to or higher than 1.3. The calculation of the tolerances and the $c_{p k}$ is repeated in various iterations to optimize the $\mathrm{Tx}_{\mathrm{i}}$. The results can be seen in table 7 of these $\mathrm{Tx}_{\mathrm{i}}$ after 3 iterations. In the results of the third iteration, not all of the $\mathrm{c}_{\mathrm{pk}}$ comply with the condition of being equal to or greater than 1.3, such that the $\mathrm{Tx}_{\mathrm{i}}$ values from the second iteration are those that should be indicated in the drawing, rather than the initial tolerances.

Table 7. Optimization of the $\mathrm{Tx}_{\mathrm{i}}$

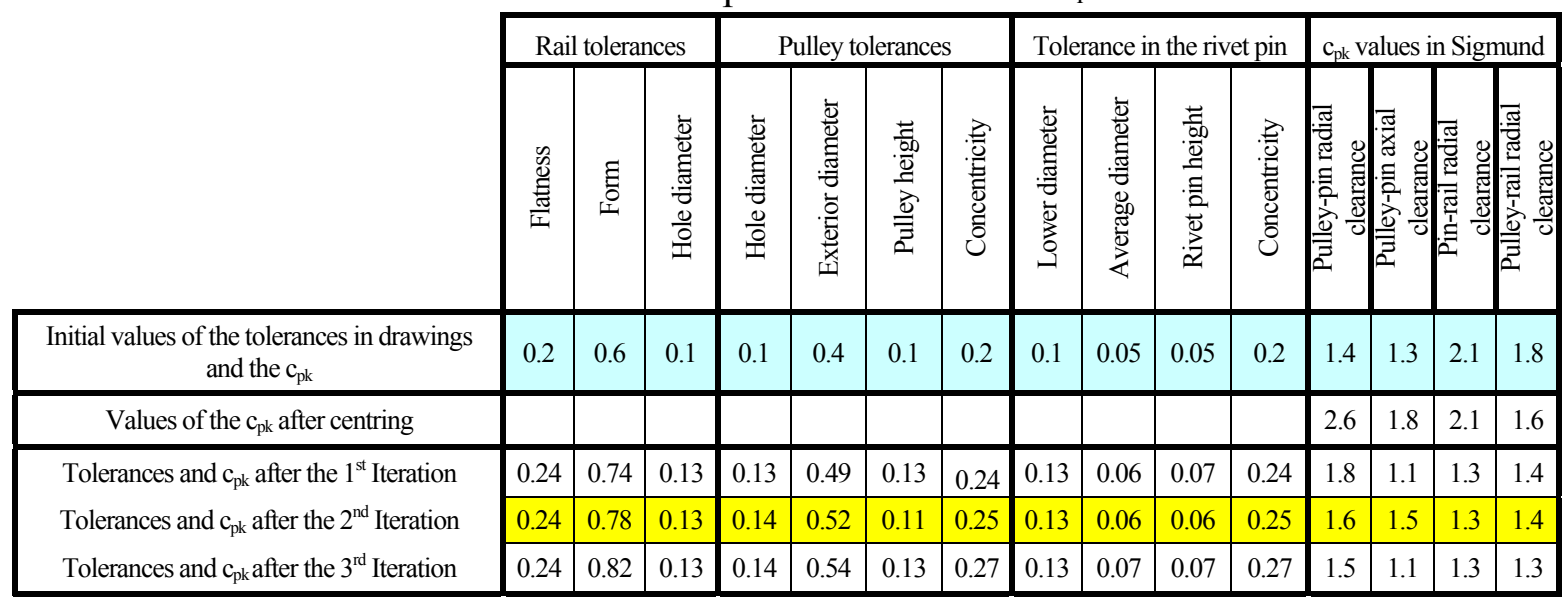


It may be seen from table 7 that all of the tolerance increased and that 8 of the 9 tolerances increased by at least $20 \%$.

\subsection{Contributions of the methodology}

The three contributions related to use of this methodology are:

- First. The design of the dimensional hierarchisation matrix (tables 1 and 3), based on the analysis of the $\mathrm{c}_{\mathrm{pk}}$ and the \%Out of each FR. This matrix takes the table proposed by Islam [14] as a reference, however that work contains no analysis of the table in terms of the $\mathrm{c}_{\mathrm{pk}}$ and the \%Out values of each FR, which we use as fundamental values for the modification and optimization of the GD\&T.

- Second. The design of the optimization algorithm $\mathrm{Tx}_{\mathrm{i}}$ in Excel has a flow diagram that is shown in figure 2. A total of 4 new algorithms were studied and designed, based on the concept of all the $\mathrm{C}_{\mathrm{pk}}$ of all the FR in a mechanism that are as low as possible, so that the tolerances are as high as possible, provided that the $\mathrm{c}_{\mathrm{pk}}$ of each FR is greater than 1.3. Some studies in the literature report cost-based analyses, such as the one by Governi [4] on the design of a genetic algorithm for the optimization of $\mathrm{Tx}_{\mathrm{i}}$ tolerances. Cost is not a known design parameter when the tolerances are indicated in the draft plans. In consequence, we propose an optimization based on the condition that, normally, $1.3<\mathrm{c}_{\mathrm{pk}}<1.5$.

- Third. The combination of the three tools: CAT 3D, matrix hierarchisation and optimization algorithm. The combination of these three tools yields the tolerances, $\mathrm{Tx}_{\mathrm{i}}$, in the design, as shown in table 7.

The principal advantages of the use of 3D CAT software as against 1D CAT are that:

- It allows us to define any statistical distribution in the deviations of the parts. We have employed standard distributions, given that the real distributions of the manufacturing process are not available in the design.

- It permits the simulation of geometrical tolerances. Some geometric tolerances such as those of perpendicularity or parallelism provide important sensitivity coefficients and therefore important contributions that may be quantified in the 3D CAT.

- 3D CAT reports in a single simulation on the result of all the FR of the mechanism, it offers us the results of the distribution and the \% of the assemblies outside of the specification of each FT. Other important results are the contributions of each GD\&T in each FR that are unknown in the 1D CAT. If some of the GD\&T are modified that are found in the FR outside of specifications, the influence of these modifications in all of the FR can be tested in a single and new simulation.

- It means that we may simulate the behaviour of the hole-pin floats.

However, the performance of a 3D tolerance analysis simulation in assemblies requires an expert designer in the operation of 3D CAT software, as there are many variables that should be controlled, involving many important decisions, such as the selection of the points in the different features that will move in the simulation.

The dimensional hierarchization matrix has been specifically designed, taking into account that it will be used together with 3D CAT software. This matrix has the objective of presenting all the necessary information in a clear way to carry out the tolerance analysis in assemblies. The results of all the FR of the mechanism are visible in this matrix, as well as all the tolerances of the parts of the mechanism, which allows easy identification of the tolerances that influence each of the FR. 
The optimization algorithm directly obtains the data from the dimensional hierarchization matrix, copying and pasting the data in Excel. This algorithm allows us to optimize the tolerances so that they are as large as possible and so that the $\mathrm{c}_{\mathrm{pk}}$ values are higher than 1.3.

To conclude this study, we may say that the joint use of the dimensional hierarchization matrix, complemented with the use of a $3 \mathrm{D}$ tolerance analysis programme and the tolerance optimization algorithm resolves many of the methodological limitations previously presented.

\section{Conclusions}

The joint use of 3D CAT software, the dimensional hierarchization matrix and an optimization algorithm, consecutively permits:

- 3D simulation of all the FR of a mechanism, on the basis of the initially proposed GD\&T in each piece in the assembly. As the most relevant data, it generates the limit values of the $\mathrm{FR}$, the $\mathrm{c}_{\mathrm{pk}}$ of each $\mathrm{FR}$, the \% of the FR outside specifications and the contributions.

- Identification in a single spread sheet (dimensional hierarchization matrix) of the FR that are non-compliant, and the parts and the GD\&T that influence these FR. This matrix also allows us to identify the GD\&T that should be modified, as it lists the GD\&T with contributions of over $25 \%$. The matrix therefore offers, in an easy way, the results of the 3D CAT simulation.

- Optimization of the GD\&T, searching for the $\mathrm{c}_{\mathrm{pk}}$ values that are between 1.3 and 1.5, if possible. This process, involving the joint use of the three aforementioned tools, may be rapidly repeated as many times as necessary, with the objective of optimizing the tolerance thresholds.

The use of this method, in the first stages of a design, can imply a significant saving of time and money in later stages.

\section{References}

[1] Shah, JJ, Ameta, G, Shen, Z, Davidson, J (2007). Navigating the tolerance analysis maze. Computer-Aided Design \& Applications, 4(5), pp 705-718.

[2] Wu, F, Dantan, J.-Y, Etienne, A, Siadat, A, Martin, P (2009). Improved algorithm for tolerance allocation based on Monte Carlo simulation and discrete optimization. Computers and Industrial Engineering 56(4), pp 1402-1413.

[3] Wayne Cai (2008). A new tolerance modeling and analysis methodology through a twostep linearization with applications in automotive body assembly. Journal of Manufacturing Systems 27, pp 26-35.

[4] Governi L, Furferi R, Volpe Y (2012). A Genetic Algorithms-based Procedure for Automatic Tolerance Allocation Integrated in a Commercial Variation Analysis Software. Journal of Artificial Intelligence, vol.5, $\mathrm{n}^{\mathrm{o}} 3$, pp 99-112.

[5] Chase K.W. \& Greenwood W. H. (1988). Design Issues in Mechanical Tolerance Analysis. ADCATS Report No. 87-5. Reprinted from Manufacturing Review, ASME, vol 1, no 1, pp. $50-59$.

[6]. Gao J, Chase KW and Magleby SP (1995). Comparison of Assembly Tolerance Analysis by the Direct Linearization and Modified Monte Carlo Simulation Methods. Proceedings of the ASME Design Engineering Technical Conferences Boston, MA, vol. 1, pp 350-360.

[7]. Cvetko R, Chase KW, Magleby SP (1998). New metrics for evaluating Monte Carlo tolerance analysis of assemblies. Proceedings of the ASME International Mechanical Engineering Conference and Exposition. Mechanical Engineering Department Brigham Young University, Provo UT. 
[8] Kane VE (1986). Process Capability Indices. Journal of Quality Technology, 18(1), pp 41-52.

[9] American Society of Mechanical Engineers. ASME Y14.5M-2009. Dimensioning and Tolerancing. New York.

[10] ISO, International Organization for Standardization ISO 1101:2004. Geometrical Product Specifications (GPS). Geometrical tolerancing. Tolerances of form, orientation, location and run-out.

[11] Lu C, Fuh JYH, Wong YS (2006). Evaluation of product assemblability in different assembly sequences using the tolerancing approach. International Journal of Production Research.Vol. 44, No. 23, pp 5037-5063.

[12] Ramos B, Cristóbal A, Melgosa C. (2015) Validation of a tolerance analysis simulation procedure in assemblies. Int J Adv Manuf Technol 76:1297-1310

[13] Polini W (2012). Taxonomy of models for tolerance analysis in assembling. International Journal of Production Research Vol. 50, No. 7, pp 2014-2029.

[14] Islam MN (2004). Functional dimensioning and tolerancing software for concurrent engineering applications. Computers in Industry 54, pp 169-190.

[15] Swift KG, Booker JD (2003). Process selection. From design to manufacture. Editorial: Butterworth Heinemann. $2^{\text {nd }}$ Edition, 316pp. Oxford.

[16] ISO 2768-1:1989 General tolerance. Part 1: Tolerances for linear and angular dimensions without individual tolerance indications.

[17] ISO 2768-2:1989 General tolerance. Part 2: Geometrical tolerances for feature without individual tolerance indications.

[18] ISO 8062-3:2007. Geometrical Product Specifications (GPS). Dimensional and geometrical tolerances for moulded parts. Part 3: General dimensional and geometrical tolerances a machining allowance for casting.

[19] Ngoi BKA, Lim LEN, Ong AS and Lim BH (1999). Applying the Coordinate Tolerance System to Tolerance Stack Analysis Involving Position Tolerance. The International Journal of Advanced Manufacturing Technology 15(6), pp 404-408.

[20] Shen Z, Shah JJ, Davidson JK (2008). Automatic generation of min/max tolerance charts for tolerance analysis from CAD models. International Journal of Computer Integrated Manufacturing 21 (8), pp. 869-884. 